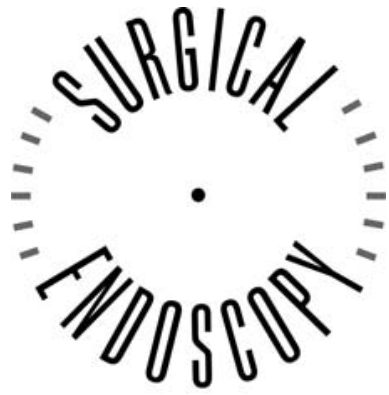

and Other Interventional Techniques

\title{
Exploring new technologies to facilitate laparoscopic surgery: creating intestinal anastomoses without sutures or staples, using a radio-frequency-energy-driven bipolar fusion device
}

\author{
J. F. Smulders, ${ }^{1}$ I. H. J. T. de Hingh, ${ }^{1}$ J. Stavast, ${ }^{2}$ J. J. Jackimowicz ${ }^{1}$ \\ ${ }^{1}$ Department of Surgery, Catharina Hospital Eindhoven, Michelangelolaan 2, 5623 EJ, Eindhoven, The Netherlands \\ ${ }^{2}$ Department of Pathology, Elisabeth Hospital Tilburg, Eindhoven, The Netherlands
}

Received: 15 October 2006/Accepted: 16 October 2006/Online publication: 19 May 2007

\begin{abstract}
Introduction: Intestinal anastomotic healing requires apposition of the collagencontaining submucosal layers of the opposing intestinal walls, which is traditionallyachieved by staples or sutures. Recently, a feedbackcontrolled bipolar sealingsystem (LigaSure) has been successfully introduced to seal and transect vessels. Since this technology depends on fusion of collagen fibres which are abundantlypresent in the intestinal wall, the possibility to create intestinal anastomoses usingthis technology was investigated in the present study.

Materials and methods: For this purpose a newgeneration radiofrequency(RF) generator and a prototype of the Ligasure Anastomotic Device (LAD) have beendeveloped. The generator incorporates a closed loop control system which monitorstissue fusion, compares it with a mathematical model of ideal fusion based on thedensity and compliance of intestinal tissue and adjusts energy output accordingly. Intotal 8 anastomoses were created in a porcine model (4 pigs, 2 anastomoses each)and healing was assessed by macroscopic and histological examination.

Results: All seals were macroscopic intact both immediate after creation and atsacrifice at the 7 th postoperative day. Between operations, pigs appeared healthy andhad normal intestinal passage. Histological examination of the anastomoses revealedundisturbed healing with granulation tissue, newly synthesised collagen in thesubmucosa and re-epithelialization at the borders of the seals. Conclusion: These results confirm the feasibility to create experimental intestinalanastomoses using LigaSure technology. This may be an important step towards thedevelopment of new laparoscopic equipment combining dissecting and reconstructiveproperties within one single instrument.
\end{abstract}

Key words: LigaSure - Intestinal anastomosis Experimental animal — Laparoscopy

Complex laparoscopic gastrointestinal surgery typically requires division of the mesentery with its multiple blood vessels and the intestine itself. Thereafter, an intestinal anastomosis has to be performed in cases where restoration of intestinal continuity is indicated. For these purposes, a multitude of dedicated laparoscopic instruments has been developed in recent decades, using electrocoagulation, ultrasonic energy, clips, sutures, or staples to obtain hemostasis and sealing of intestinal segments [5]. Unfortunately, none of the available instrumentation is capable of both tissue dissection and anastomotic reconstruction in an efficient way. This means that laparoscopic equipment has to be changed numerous times in the course of a procedure which prolongs operative time, increases costs, and adds to the complexity of the procedure. Ideally, it should be possible to complete an entire procedure laparoscopically using only a single dedicated instrument.

Recently, a feedback-controlled bipolar sealing system or LigaSure (Tyco Healthcare, Valleylab, Boulder, $\mathrm{CO})$ has been added to the armamentarium of the laparoscopic surgeon. This device applies a precise amount of mechanical pressure and radiofrequency energy to tissue, causing fusion of apposing layers by creating a seal of denatured collagen and elastin fibers. This seal can than be transected, permitting the safe division of a multitude of tissues, including blood vessels with a diameter of up to $7 \mathrm{~mm}$ [2]. Since its introduction, the LigaSure device has indeed been proven beneficial in laparoscopic surgery, but at present it is used almost exclusively to divide tissues, especially the mesentery $[6$, 8, 17, 18].

The LigaSure technique acts by sealing collagencontaining tissue layers [5], and because collagen, a 


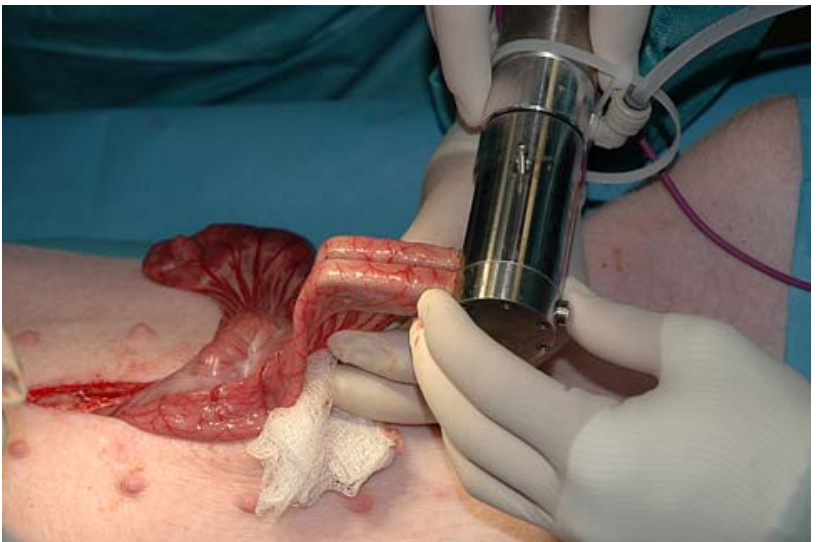

Fig. 1. Both jaws of the LigaSure anastomotic device are placed in the intestinal lumen and closed at the antimesenteric side. This is the situation immediately prior to the administration of radiofrequency energy.

protein, is abundantly present in the intestinal wall [16], the technique should theoretically be capable of creating intestinal sealing lines and thus intestinal anastomoses.

This possible application would greatly contribute to the ideal of performing complex laparoscopic gastrointestinal surgery with a single laparoscopic instrument, because it would combine the proven efficient dissecting properties of LigaSure technology with new reconstructive capacities. Therefore, in this experimental study, the feasibility of creating intestinal anastomoses using a prototype of the LigaSure anastomotic device (LAD) was investigated in an animal model.

\section{Materials and methods}

\section{LigaSure anastomotic device}

A prototype of the LigaSure anastomotic device (LAD) and a newgeneration radio-frequency ( $\mathrm{RF}$ ) generator were kindly provided by Valleylab (Boulder, CO) for the duration of the experimental study. The generator incorporates a closed-loop control system that monitors tissue fusion, compares it with a mathematical model of ideal fusion based on the density and compliance of intestinal tissue, and adjusts energy output accordingly, as previously described [13]. With the LAD both RF-energy and mechanical pressure can be applied by pulling the intestinal wall between the jaws of the device and closing it (Fig. 1).

\section{Operative procedure}

Approval of the local ethics committee was obtained before the start of the experimental study, which was performed in four female adult domestic pigs. Animals were denied solid food for $24 \mathrm{~h}$ before the start of the experiment, but they had free access to water. Premedication included Ketamine $(20 \mathrm{mg} / \mathrm{kg})$ and Acepromazine $(0.5 \mathrm{mg} / \mathrm{kg})$.

Animals were anesthetized with pentobarbitone $(6 \mathrm{mg} / \mathrm{kg})$ and isoflurane (2\% in a mixture of oxygen). After a single dose of prophylactic antibiotics was administered, a median laparotomy was performed. In each pig, two functional side-to-side anastomoses were performed in the distal ileum, $50 \mathrm{~cm}$ and $20 \mathrm{~cm}$ proximal to the ileocecal valve, respectively. This was achieved by taking a loop of small bowel at the designated area and opening both intestinal lumina at the top of the loop. After placing a traction suture to align the lumina, a jaw of the LAD was placed in each of the two opposing intestines and mechanical pressure on the intestinal walls was achieved by closing the jaws antimesenterically (Fig. 1). After perfect positioning of the LAD was assured, radiofrequency energy was applied by activating the RF-generator. The combination of mechanical pressure and radiofrequency energy causes collagen situated in the intestinal wall to denature and seals the apposing intestinal walls, where they are situated within the jaws of the LAD. After removal of both jaws of the LAD the seal was inspected for completeness, and the area was inspected for signs of thermal spread or other side-effects. The seal then was transected in the middle using scissors to create a wide anastomosis. Finally, the anastomosis was completed by closing the top of the loop with conventional interrupted sutures. Thus, each anastomosis consisted of two essentially different parts, one an experimental sealed fusion line in the side-to-side fusion and the other a conventional sutured line at the top of the anastomosis.

Animals were allowed oral intake from the first postoperative day and were examined on a daily basis for rectal temperature and bowel sounds until sacrifice at postoperative day (POD) 7. On this day animals were re-anesthetized as previously described and a relaparotomy was performed. The abdomen was inspected for signs of peritonitis and anastomotic leakage, and both anastomoses were identified. Finally, the two intestinal segments containing the anastomosis in the middle were removed from the animal, fixed in formalin and sent to the pathology department for further analysis.

\section{Histology}

After fixation, the anastomotic site was lamelleted from top to bottom. Each lamella was completely enclosed, enabling examination of both the sealed and the sutured part of the anastomosis in a single slide. After dehydration and imbedding in paraffin the blocks were cut in slices of $3 \mu \mathrm{m}$. Then the slides were stained with hematoxylin-eosin by Mayer (deparaffination; wash in demiwater; hematoxylin by Mayer, $5 \mathrm{~min}$; wash with streaming water, $5 \mathrm{~min}$; eosin $0.5 \%, 5 \mathrm{~min} ; 3$ dips into a water bath; dehydration) or Sirius-red (deparaffination; wash in streaming aquadest; incubation with Sirius-red solution $(\mathrm{pH}=2), 60 \mathrm{~min}$; wash with $0.01 \mathrm{~N} \mathrm{HCl}, 2$ min; dehydration). Histological assessment of all slides was performed by an experienced pathologist.

\section{Results}

\section{Surgical technique}

No adverse events were observed during construction of the anastomoses. Upon completion, all anastomoses appeared macroscopically intact without signs of thermal spread. Three of the animals showed normal behavior, food intake, and intestinal function almost immediately after the operation and during the course of the experiment. In one pig a mild fever $\left(38^{\circ} \mathrm{C}\right)$ and signs of ileus were noted, but only on the first postoperative day.

At re-laparotomy no signs of ileus, generalized peritonitis, or extraluminal bowel contents were noted. Upon visual inspection, seven anastomoses were completely intact and appeared to have functioned normally. The top of one of the anastomoses showed signs of a localized peritonitis (adhesions, fibrous tissue), apparently caused by a small and self-limiting leak from the 


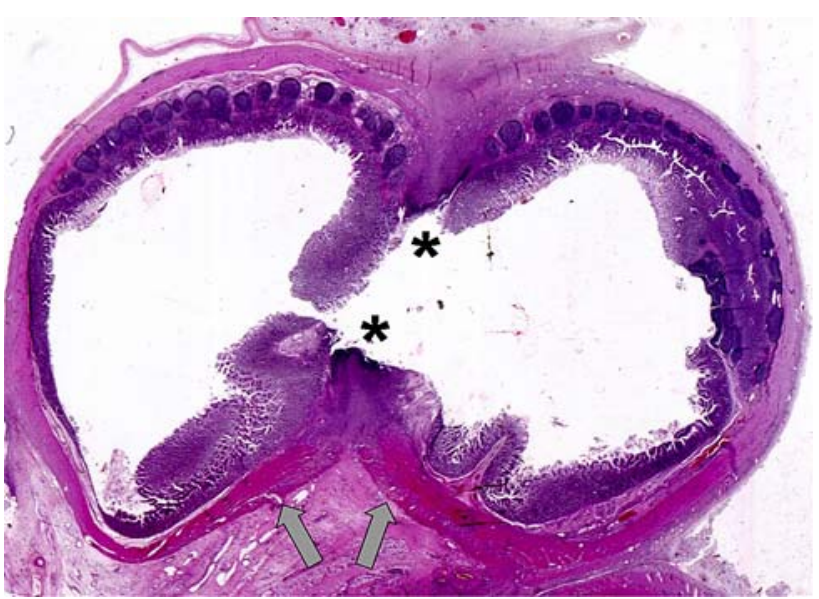

Fig. 2. Cross section of a sealed anastomosis immediately after construction (H\&E-staining, magnification 10×). Sealed areas are identified $(*)$. Adequate alignment of the muscularis propria is clearly visible (gray arrows).

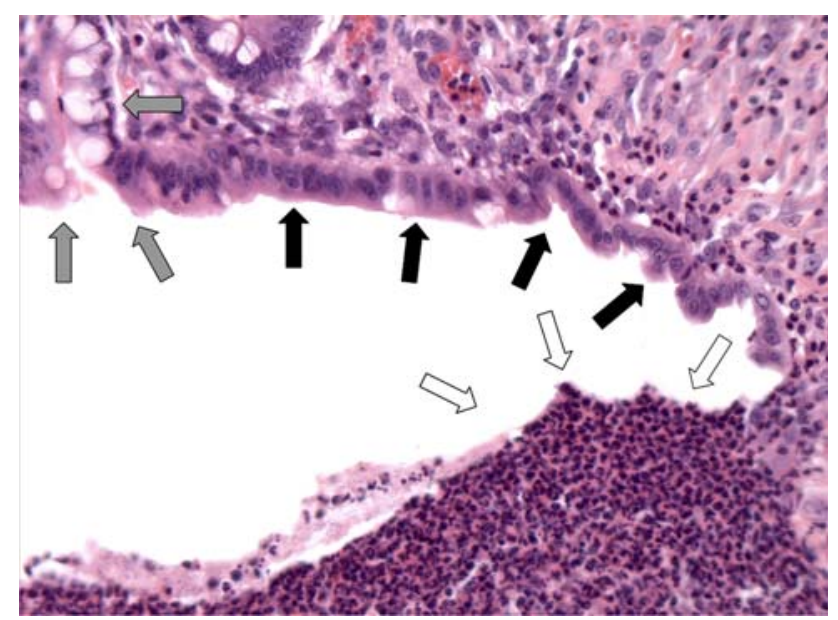

Fig. 3. Slide (H\&E staining, magnification $200 \times$ ) showing the surface of the sealed anastomosis with granulation tissue containing a mixed infiltrate (open arrows), normal mucosal lining adjacent to the seal (gray arrows), and re-epithelialization by young epithelial cells covering the sealed area (black arrows).

sutured line. This anastomosis was found in the animal suffering from transient postoperative fever and ileus.

\section{Histology}

Hematoxylin-eosin slides show that the luminal side of the intestine adjacent to both the sealed and the sutured anastomoses is covered with intestinal epithelium with spared crypt-villi architecture (Fig. 2). The lamina propria has a normal cellularity and the muscularis mucosa and muscularis propria are normally formed. In the submucosa, lymphfollicular hyperplasia with formation of germinal centers was noted. These are all normal characteristics of the intestinal wall. At the anastomotic sites evident changes are present (Fig. 3). Along the sealed part of the anastomosis, the mucosa has disappeared and is replaced by a mixed inflammatory infil-

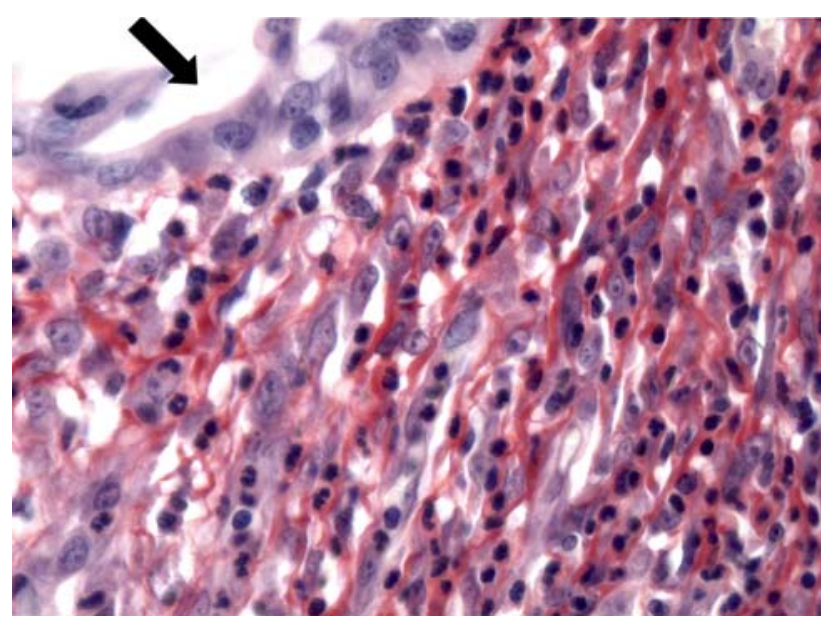

Fig. 4. Slide (Sirius red staining, magnification $400 \times$ ) showing loosely arranged, newly formed collagen as thin red fibers in the submucosal sealed area. Fibers are orientated in a longitudinal fashion, an early sign of organization. Again, re-epithelialization is noted at the luminal side of the seal (black arrow).

trate of lymphocytes, granulocytes, and plasma cells mixed with young fibroblasts and beginning vascular proliferation, all clear features of granulation tissue. Interestingly, growth of new epithelium is present at the borders of this granulation tissue, and because this epithelium grows toward the center of the defect, this is a clear feature of restoration of the mucosal layer. Underneath this inflammatory zone, the muscularis propria of both intestinal sides is parallel aligned, and no specific changes are seen in the subserosal fat.

In contrast to the sealed part of the anastomoses, granulation tissue is not displayed in the sutured parts. Instead, signs of acute inflammation with the beginning of abscess formation and foreign body giant cell reaction are apparent, especially around the areas where the sutures are situated.

Sirius-red stained slides show the formation of new thin collagen fibers in the submucosal area of both the sealed and the sutured parts of the anastomoses (Fig. 4). The fibers are only loosely arranged, but focally they already show the beginning of organization, especially in the sealed anastomoses. Although histological assessment does not allow for quantification of the collagen present, the general impression was that more collagen was formed underneath the sealed parts of the anastomoses than between the sutured parts.

These histological findings were remarkably similar and constant in seven of the anastomoses, the only exception being the anastomosis in which minor leakage was diagnosed at re-laparotomy. In that anastomosis an exaggerated inflammatory response and luminal spill was seen along the outside border of the sutured anastomotic part, confirming leakage at the site.

\section{Discussion}

In this study, the feasibility of constructing intestinal anastomoses by using a combination of radiofrequency energy and mechanical pressure was confirmed, at least 
in an experimental animal model. For this purpose a prototype LigaSure anastomotic device was developed, which functioned adequately throughout the course of the study without unforeseen side-effects. Anastomotic healing was assessed by qualitative histological and macroscopical examination and evaluation of function.

Physiological wound healing has been defined as the highly regulated process of cellular, humoral, and molecular events activated at the time of injury and resulting in a time-dependent but predictable and orderly pattern of tissue repair [3]. Three successive phases can be recognized in this process by histological examination: inflammation, proliferation, and tissue remodeling [15]. Previous experimental studies have proven that the inflammatory and proliferative phases are most intense during the first postoperative week, and consequently this period is deemed crucial for uneventful anastomotic healing [19]. Therefore, all LAD-constructed anastomoses were assessed by an experienced pathologist at POD 7. This revealed the presence of an inflammatory process around the sealed anastomotic tissues. In addition, signs of proliferation were profusely present, such as reepithelialization to restore the mucosal barrier, formation of new collagen fibers in the submucosa to regain anastomotic strength, and neovascularization to restore blood supply. Therefore, it is concluded that LAD-constructed anastomoses display features of physiological intestinal anastomotic healing.

Macroscopical inspection, both immediately after construction as well as at POD 7, revealed that all sealed parts of the anastomoses were completely intact. One case of minor anastomotic leakage was diagnosed, but this appeared to be caused by a small disruption of the sutured anastomotic part. Adequate functioning of the anastomoses was deduced from the observation that animals displayed normal behavior, food-intake, bowel sounds, and passage of stools in the postoperative period. Moreover, no signs of ileus were noted at re-laparotomy. Taken together, histological, macroscopical, and functional parameters all confirmed undisturbed and successful healing of the sealed anastomotic tissues. However, before application of this technology can be considered in clinical practice, these parameters should be investigated in a larger number of animals and at different time points throughout the healing process. Besides this, additional functional parameters such as, for instance, the development of anastomotic strength should be investigated. Since its introduction in surgical practice, LigaSure technology has been proven to significantly save operative time and costs and to reduce the complexity of operative procedures $[6,8,17,18]$. Interestingly, these advantages are almost exclusively achieved by using it as a dissecting tool to take down the intestinal mesentery, even though the presence of collagen in many other tissues renders this technology potentially suitable for numerous other applications. Experience in other organs is currently limited to a few clinical and experimental studies that have investigated the possibility of sealing and transecting collagen-containing tissues, such as the cystic duct [12], ureter [4], lung parenchyma $[11,14]$, liver [7, 10], hemorrhoids [1,9], and appendix
[20]. The present study differs fundamentally from these studies since the LigaSure was not applied for its dissecting properties but for its possible reconstructive capacities, the creation of intestinal anastomoses. This application may be particularly helpful for the laparoscopic gastrointestinal surgeon as the creation of an intestinal anastomosis using laparoscopic instruments is considered technically demanding, costly, time-consuming, and complex because of the numerous changes of instruments required. Many surgeons even prefer to perform a small laparotomy in the reconstructive phase to create an anastomosis outside of the abdomen, thereby potentially compromising the benefits of laparoscopic surgery. The availability of a laparoscopic application of the LAD may overcome these difficulties and facilitate laparoscopic gastrointestinal surgery in the future.

The ideal laparoscopic equipment should combine dissecting as well as reconstructive capacities within a single instrument and without the need to reload it after each application. The current experimental finding that LigaSure technology may be applied to construct intestinal anastomoses should be regarded as a promising step towards the development of such an instrument, warranting further research into this subject.

\section{References}

1. Basdanis G, Papadopoulos VN, Michalopoulos A, Apostolidis S, Harlaftis N (2005) Randomized clinical trial of stapled hemorrhoidectomy vs open with LigaSure for prolapsed piles. Surg Endosc 19: 235-239

2. Carbonell AM, Joels CS, Kercher KW, Matthews BD, Sing RF, Heniford BT (2003) A comparison of laparoscopic bipolar vessel sealing devices in the hemostasis of small-, medium-, and largesized arteries. J Laparoendosc Adv Surg Tech A 13: 377-380

3. Dubay DA, Franz MG (2003) Acute wound healing: the biology of acute wound failure. Surg Clin North Am 83: 463-481

4. Goldstein SL, Harold KL, Lentzner A, Matthews BD, Kercher KW, Sing RF, Pratt B, Lipford EH, Heniford BT (2002) Comparison of thermal spread after ureteral ligation with the LaparoSonic ultrasonic shears and the LigaSure system. J Laparoendosc Adv Surg Tech A 12: 61-63

5. Harrell AG, Kercher KW, Heniford BT (2004) Energy sources in laparoscopy. Semin Laparosc Surg 11: 201-209

6. Heniford BT, Matthews BD, Sing RF, Backus C, Pratt B, Greene FL (2001) Initial results with an electrothermal bipolar vessel sealer. Surg Endosc 15: 799-801

7. Horgan PG (2001) A novel technique for parenchymal division during hepatectomy. Am J Surg 181: 236-237

8. Howard TJ, Mimms S (2005) Use of a new sealing device to simplify jejunal resection during pancreaticoduodenectomy. Am J Surg 190: 504-506

9. Lawes DA, Palazzo FF, Francis DL, Clifton MA (2004) One year follow up of a randomized trial comparing LigaSure with open haemorrhoidectomy. Colorectal Dis 6: 233-235

10. Romano F, Franciosi C, Caprotti R, Uggeri F, Uggeri F (2005) Hepatic surgery using the LigaSure vessel sealing system. World J Surg 29: 110-112

11. Santini M, Vicidomini G, Baldi A, Gallo G, Laperuta P, Busiello L, Di Marino MP, Pastore V (2006) Use of an electrothermal bipolar tissue sealing system in lung surgery. Eur J Cardiothorac Surg 29: 226-230

12. Schulze S, Krisitiansen VB, Fischer HB, Rosenberg J (2002) Sealing of cystic duct with bipolar electrocoagulation. Surg Endosc 16: $342-344$ 
13. Shields CE, Schechter DA, Tezlaf P, Baily AL, Dycus S, Cosgriff N (2004) Method for creating ideal tissue fusion in soft-tissue structures using radio frequency energy (RF). Surg Technol Int 13: 49-55

14. Shigemura N, Akashi A, Nakagiri T, Ohta M, Matsuda H (2004) A new tissue sealing technique using the LigaSure system for nonanatomical pulmonary resection: preliminary results of sutureless and stapleless thoracoscopic surgery. Ann Thorac Surg 77: $1415-1418$

15. Steed DL (2003) Wound-healing trajectories. Surg Clin North Am 83: $547-555$

16. Stumpf M, Klinge U, Wilms A, Zabrocki R, Rosch R, Junge K, Krones C, Schumpelick V (2005) Changes of the extracellular matrix as a risk factor for anastomotic leakage after large bowel surgery. Surgery 137: 229-234
17. Takada M, Ichihara T, Kuroda Y (2005) Comparative study of electrothermal bipolar vessel sealer and ultrasonic coagulating shears in laparoscopic colectomy. Surg Endosc 19: 226-228

18. Targarona EM, Balague C, Marin J, Neto RB, Martinez C, Garriga J, Trias M (2005) Energy sources for laparoscopic colectomy: a prospective randomized comparison of conventional electrosurgery, bipolar computer-controlled electrosurgery and ultrasonic dissection. Operative outcome and costs analysis. Surg Innov 12: 339-344

19. Witte MB, Barbul A (2003) Repair of full-thickness bowel injury. Crit Care Med 31: S538-S546

20. Yang HR, Wang YC, Chung PK, Jeng LB, Chen RJ (2005) Laparoscopic appendectomy using the LigaSure vessel sealing system. J Laparoendosc Adv Surg Tech A 15: 353-356 